\title{
Comparative DNA Flow Cytometric Study of Primary Intraocular and Central Nervous System Lymphomas
}

\author{
Teresa Martinu*, MDCM, Claudia P. Correia, MD, \\ Andersson M. Figueiredo, MD, Miguel N. Burnier Jr., MD, PhD
}

\begin{abstract}
Primary intraocular lymphoma is generally considered as a subset of primary CNS lymphoma. This study attempts to show that they may in fact represent distinct entities by comparing their respective proliferation rates using DNA flow cytometry. Four samples of primary intraocular lymphoma and seven samples of primary CNS lymphoma were analyzed, all from paraffin-embedded tissue. All tumors were of the large B-cell type. A normal human tonsil sample was used as a control. Tissue samples were analyzed by DNA flow cytometry, which is a precise and objective method to measure DNA content and cell proliferation of a tumor. S-phase fraction (SPF) and DNA content were measured for each sample. The average SPF for primary intraocular lymphoma was significantly higher than that of primary CNS lymphoma, 23.8 (range: 18.9 to 29.6) versus 15.1 (range: 1.1 to 25.1) respectively. Of the 11 tumors analyzed, 2 brain tumors were aneuploid and 1 eye tumor was peridiploid. All other tumors were diploid. Thus, no significant pattern was detected in the DNA content of the tumors. This lack of clinical significance of tumor aneuploidy is consistent with data reported in the literature. The results of this study indicate that primary intraocular lymphoma is more aggressive and of higher grade than primary CNS lymphoma. The different proliferation rates of intraocular and CNS lymphomas may be explained by either their different spatial location or a distinct genetic composition, the latter reinforcing the hypothesis that the two are fundamentally different entities
\end{abstract}

\section{INTRODUCTION}

Primary intraocular and central nervous system (CNS) lymphomas are defined as extranodal nonHodgkin's lymphomas that are not associated with systemic involvement at the time of diagnosis $(1,2,3,4)$. They are rare and lethal neoplasms that represent a challenge in terms of their diagnosis, treatment, and prognosis. Their terminology, classification, and pathogenesis are a source of continuous confusion. The research presented in this report attempts to elucidate the pathogenesis of primary intraocular and CNS

* To whom correspondence should be addressed: Teresa Martinu, MD, Division of Pulmonary and Critical Care Medicine, Duke University Medical Center, DUMC Box 3221, Durham, NC, 27710. Tel: 919-684-8111. Email: tereza.martinu@duke.edu lymphomas by comparing their DNA flow-cytometric profiles. Although intraocular lymphomas are generally considered as a subset of CNS lymphomas, this study attempts to show that they are actually different entities, of different genetic origin and pathogenesis.

Primary intraocular and CNS lymphomas combined represent about $2 \%$ (5) to $5 \%$ (6) of all lymphomas. Their incidence is higher in immunocompromised patients, notably those with AIDS, transplanted organs, and congenital immunodeficiencies $(1,3,7)$. These neoplasms most often occur after 60 years of age $(5,8)$.

Histopathologically, most primary intraocular and CNS lymphomas are high-grade large B-cell lymphomas (Figs 1A,B,C) (2,9,10). but T-cell lymphomas have also been reported (1). The malignant 

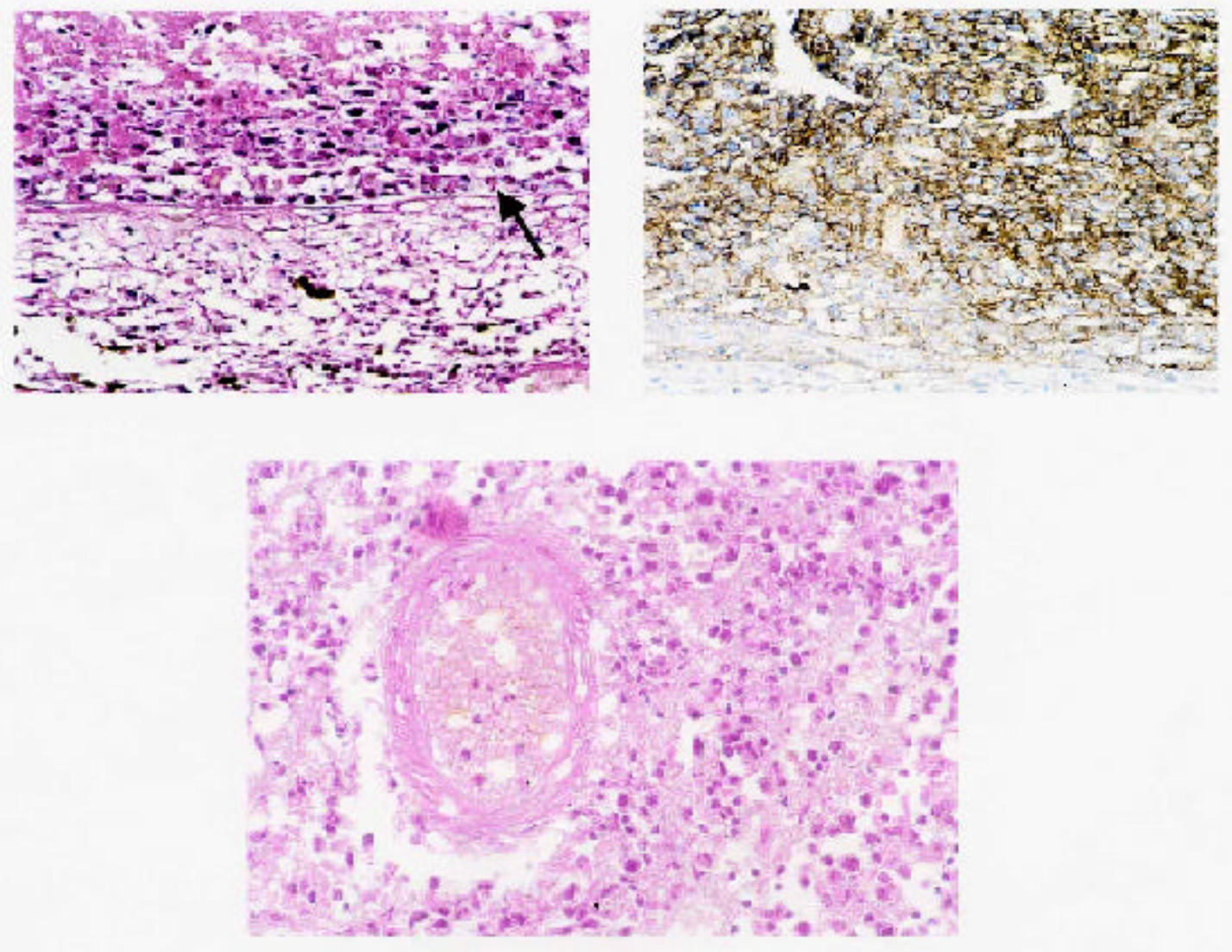

Figure 1. Histopathological and immunohistochemical features of primary large B-cell lymphoma of the retina and CNS. A. H\&E stain. Primary intraocular lymphoma showing large viable and necrotic neoplastic cells internal to the Bruch's membrane. Small reactive lymphocytes are present external to the Bruch's membrane.

B. L-26 monoclonal antibody marker. Primary intraocular lymphoma showing large B-cells in the retina.

C. H\&E stain. Primary CNS lymphoma showing a blood vessel with neoplastic cells demonstrating a perivascular cuffing pattern.

cells of large B-cell lymphomas characteristically have abundant cytoplasm, round indented nuclei with large single or multiple nucleoli, and prominent nuclear membranes. Mitotic figures are common and extensive areas of central hemorrhagic necrosis are often apparent. Malignant cells are visible in the parenchyma, characteristically accumulated around blood vessels. Benign reactive T-lymphocytes often surround the neoplastic lesions $(11,12)$.

Early intraocular lymphoma lesions occur characteristically between the retina and Bruch's membrane $(2,9,12,13)$. Although it sometimes occurs as solely a malignancy of the eyes, $50 \%$ to $80 \%$ of patients who present with primary intraocular lymphoma exhibit CNS involvement later in their disease. Conversely, intraocular involvement is present in 12 to 18 of patients with primary CNS lymphoma $(1,2,14)$. It is interesting to note that primary intraocular lymphoma is more likely to be followed by
CNS involvement, rather than vice versa. The eyes are involved bilaterally in up to $75 \%$ of cases with or without concomitant brain involvement $(2,8,9,10)$.

Primary intraocular lymphoma presents as corticosteroid-resistant uveitis, vitritis, or chorioretinitis, $(2,7,10,15)$ with initial symptoms of blurred vision and floaters $(13,14)$. Diagnostic techniques include vitrectomy, biopsy, or enucleation. Primary CNS lymphoma often presents with multiple lesions which can be located anywhere in the CNS, including the spine and leptomeninges, and which usually give rise to non-specific neurologic symptoms $(1,14,16,17)$. It is diagnosed by imaging and biopsy. Primary CNS lymphoma is currently treated with chemotherapy and adjunctive cranial radiotherapy. Bilateral ocular and whole brain irradiation are used for intraocular involvement. With optimal treatment, the median survival has been reported to be 30 to 45 months in non-immunocompromised patients with primary 

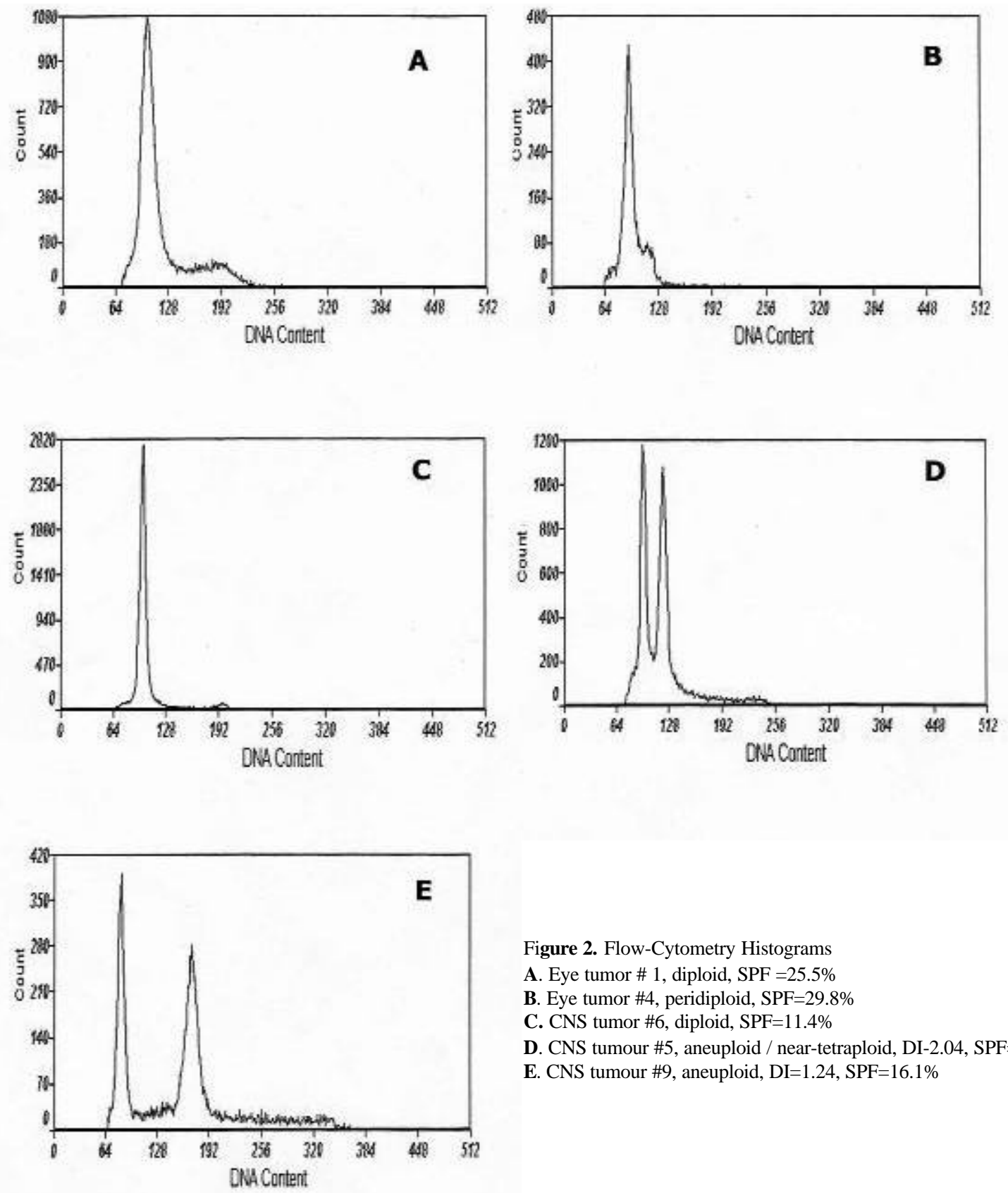

Figure 2. Flow-Cytometry Histograms

A. Eye tumor \# 1, diploid, $\mathrm{SPF}=25.5 \%$

B. Eye tumor \#4, peridiploid, $\mathrm{SPF}=29.8 \%$

C. CNS tumor \#6, diploid, $\mathrm{SPF}=11.4 \%$

D. CNS tumour \#5, aneuploid / near-tetraploid, DI-2.04, SPF=32.6\%

E. CNS tumour $\# 9$, aneuploid, $\mathrm{DI}=1.24, \mathrm{SPF}=16.1 \%$

CNS lymphoma (4).

The pathogenesis of primary intraocular and CNS lymphomas is a continuous source of controversy. First, the origin of the malignant cells is obscure. The retina and the CNS lack a lymphatic system and intrinsic lymphocytes. Furthermore, primary intraocular and CNS lymphomas are particular in their high specificity for the central nervous system, including the retina, which is an anatomical and functional extension of the brain; systemic spread has been reported in only 7-8 of autopsies $(3,15)$. However, these systemic occurrences could represent second primaries, rather than metastases $(6,17)$. There are several theories that attempt to

explain the origin of the primary CNS lymphoma. The theory of malignant transformation within a lymphocytic reaction around a viral infection has been proposed (1). In AIDS patients, for example, involvement of the EBV virus in primary CNS lymphoma has been reported (1). Similarly, herpes virus involvement in immunocompetent patients has also been suggested $(1,16)$. Another hypothesis is that B-lymphocytes are transformed elsewhere in the body and develop specific binding molecules for the CNS. Specific receptors for these molecules on the CNS endothelial cells may explain the "homing pattern" of this neoplasm $(1,18)$. The relatively privileged sites of 


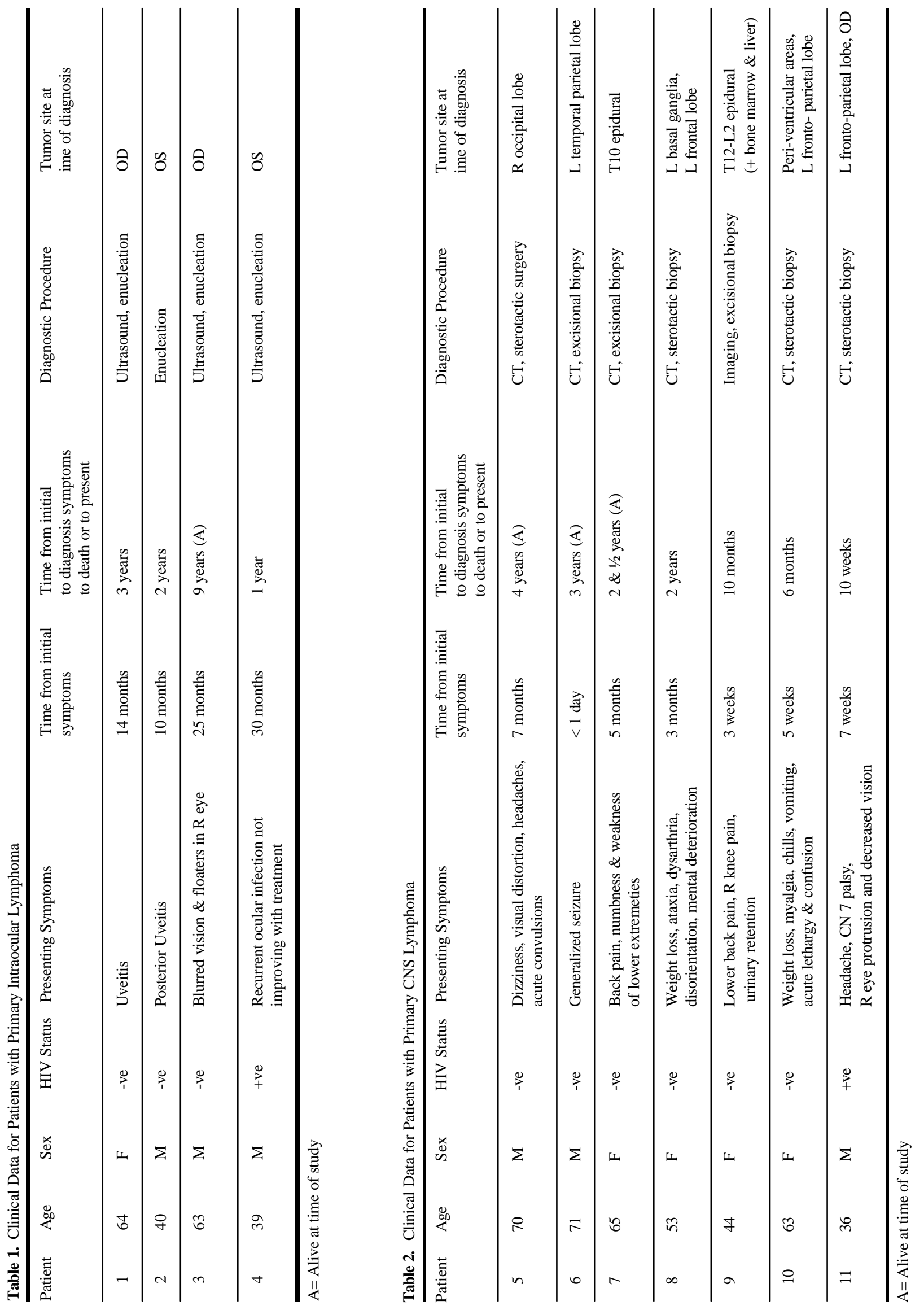


the CNS and retina, sequestered from the normal immune surveillance mechanisms, may further encourage the malignant growth $(1,7,17)$. Another possibility is that these lymphomas in fact represent metastases from occult systemic lymphomas (19). Finally, completely different precursors for the malignant lymphocytes have also been proposed: reticulum cells, microglial, and glial cells $(9,14,17)$.

Second, multifocal presentation of primary intraocular and CNS lymphomas is poorly understood; how does the tumor spread within the CNS without a communicating lymphatic system? Hematogenous spread is possible but, theoretically, it should lead to a higher occurrence of systemic metastases. Direct spread seems unlikely due to the large areas of normal brain tissue often located between lesions and the reported absence of brain involvement in bilateral intraocular lymphoma. Intraneural expansion is one possibility, although, to the best of our knowledge, the actual invasion of the optic nerve with neoplastic cells has never been demonstrated. Lastly, the choroidal circulation could be responsible for the retinal involvement (15).

To further elucidate the possible origin and mechanism of multifocal distribution of primary intraocular and CNS lymphomas, comparison of primary lymphoma of the retina with that of the CNS via DNA flow cytometry was performed.

DNA flow cytometry is an objective method for measurement of the DNA content (i.e. ploidy) and proliferative activity (determined by S-phase fraction or $\mathrm{SPF}$ ) in normal and malignant cells. The advantage of this method is the ability to measure a multitude of parameters on thousands of individual cells in a short time. Furthermore, DNA flow cytometric studies are effective with both fresh tissue and formalin-fixed paraffinized tissue (20). Aneuploid and tetraploid populations have been described for non-Hodgkin's lymphomas, although abnormal DNA content appears to have very limited clinical or prognostic significance (21). In contrast, previous studies of non-Hodgkin's lymphoma have shown a good correlation between high proliferative activity, advanced histopathological grade, aggressive clinical behavior, and poor prognosis $(21,22)$. Non-Hodgkin's lymphomas have been reported to have generally "intermediate" proliferative activities, defined by SPFs between $4 \%$ and $10 \%$ (21).

This study aims to compare the DNA flow cytometric results for primary diffuse large B-cell intraocular and CNS lymphomas and show that these neoplasms may be genetically different. To our best knowledge, flow cytometric analysis has never been applied specifically to primary intraocular or CNS lymphomas.
Table 3. DNA flow cytometric data for intraocular lymphoma

\begin{tabular}{lll}
\hline Patient & Ploidy & SPF (\%) \\
\hline 1 & Diploid & 25.5 \\
2 & Diploid & 18.9 \\
3 & Diploid & 21.2 \\
4 & Peridiploid2 & 9.8 \\
\hline Average & & 23.9 \\
\hline
\end{tabular}

\section{PATIENTS, MATERIALS AND METHODS Patients}

Four paraffinized samples of primary intraocular lymphoma, with associated clinical information, were obtained from the Federal University of San Paulo, Brazil (3 samples) and from India (1 sample).

To obtain primary CNS lymphoma samples, computerized and manually filed data, as well as individual patient charts at the Cancer Registry of the Royal Victoria Hospital and the Montreal Neurological Institute Medical Records, Montreal, were analyzed. Data for all 18 patients registered as having primary CNS lymphoma between 1995 and 1999 was collected. The pathology reports and individual histopathological slides were reviewed with experienced CNS and ophthalmic pathologists in order to confirm the diagnoses. Seven patients with confirmed primary CNS lymphoma, whose paraffin blocks were prepared after 1995 and were available at the time of the present research, were identified and used for this study. Histolpathologically, all tumors included in this study were large B-cell lymphomas.

\section{DNA Flow Cytometry}

The following steps were performed on each of the 4 ocular and 7 CNS paraffin-embedded tissue samples, similar to the method described by Riley et al. (20).

Tissue deparaffinization: Two to five 50um sections of paraffin-embedded tissue were cut. Tissue sections were incubated twice for 10 minutes in $10 \mathrm{ml}$ ofxylene. Rehydration of the tissue was performed by 10 minute incubations in decreasing concentrations ofethanol: $100 \%$ twice, $95 \%, 70 \%, 50 \%$. The tissue was then minced and left in $10 \mathrm{ml}$ of distilled water for 2-3 nights.

Tissue disaggregation and DNA staining: A fresh pepsin solution was prepared and brought to $37^{\circ} \mathrm{C}$. Water was removed from each sample and 1 to $2 \mathrm{ml}$ of pepsin was added to the tissue. Samples were vortexed and incubated for 30 minutes at $37^{\circ} \mathrm{C}$. After incubation, each sample was diluted twice with cold PBS (phosphate buffered solution) and centrifuged at $900 \mathrm{~g}$ for 10 minutes. The pellet was resuspended in $2 \mathrm{ml}$ of Krishan DNA staining buffer $(50 \mathrm{mg} / \mathrm{ml}$ propidium iodide, Img/ml trisodium citrate, 350 units RNAse, 
Table 4. DNA flow cytometric data for CNS lymphoma

\begin{tabular}{lll}
\hline Patient & Ploidy & SPF (\%) \\
\hline 5 & Aneuploid, near-tetraploid & \\
& DNA content, DI=2.04 & 32.6 \\
6 & Diploid & 11.4 \\
7 & Diploid & 1.1 \\
8 & Diploid & 25.1 \\
9 & Aneuploid & 16.1 \\
& DNA content, DI=1.24 & 8.3 \\
10 & Diploid & 11.0 \\
\hline 11 & Diploid & 15.1 \\
\hline \multirow{2}{*}{ Average } & & \\
\hline
\end{tabular}

$0.75 \mathrm{ml} / \mathrm{ml} \mathrm{NP}-40$, in distilled water), vortexed, and incubated in the dark at $4^{\circ} \mathrm{C}$ overnight. The sample was then aspirated successively through 18, 20, and 22 gauge needles, and then filtered through a 53×37 mesh.

\section{Flow cytometric analysis}

An EPICS XL flow cytometer (Beckman Coulter® Corp., USA) was used for DNA measurements. Before each set of measurements, the instrument was adjusted by the use of normal human tonsil cells. An average of 28,000 nuclei (range, 13,595 to 38,625 nuclei) were analyzed for each sample. The coefficient of variation, defined as the standard deviation in percent of the mean DNA value of the diploid Gl/G0 peak, was 5.5 mean (range, 3.5 to 8.5 ). The DNA flow cytometry data was analyzed by MultiCycle for Windows, advanced DNA cell cycle analysis software. Phoenix flow systems (San Diego, California).

\section{Definition of DNA index (DI), ploidy, and S-phase fraction}

The DI of the diploid tumor cells was defined as 1.0. Tumors were defined as diploid by the presence of a single symmetrical G0/G1 peak in the histogram. A single broad asymmetrical G0/G1 peak was observed in one case of ocular lymphoma, a phenomenon most likely due to a minor degree of aneuploidy. According to other authors, we considered it appropriate to define such a tumor as "peridiploid" (22). Tumors with more than one discrete G0/G1 peak were considered aneuploid. The degree of aneuploidy was expressed as a DI, which was defined as the ratio of the channel position of the G0/G1 aneuploid peak to the channel position of the G0/G1 of the diploid peak. A DI of 2.0 corresponds to a tetraploid population. Cell cycle distribution, including the S-phase fraction, was estimated according to the Dean and Jett polynomial Sphase model.

\section{RESULTS}

Among patients with primary intraocular lymphoma (table 1), 3 were male and 1 was female. The mean age of patients was 51.5 years (range, 39-64). One patient was HIV positive at the time of diagnosis. Patients presented with symptoms such as uveitis, blurred vision, floaters, and recurrent eye infections. The average time from initial symptoms to diagnosis was approximately 19.75 months (range, 10 to 30 ). At the time of this study, 1 patient is still alive, 9 years after initial symptoms; 3 patients were dead. The average time from initial symptoms to death is 2 years (range, 1 to 3). All patients were diagnosed by ultrasound or enucleation. There is no reported brain or systemic involvement.

Regarding patients with primary CNS lymphoma (table 2), 3 were male and 4 female. The mean age was 57.43 years (range, 36-70). One patient was HIV positive at the time of diagnosis. Patients presented with symptoms such as weight loss, flu-like symptoms, headache, back pain, muscle pain, vague neurological symptoms, and focal neurological deficits. The average time from initial symptoms to diagnosis was approximately 2.5 months (range, 1 day to 7 months). At the time of this study, 3 patients are still alive, 4, 3, and 2.5 years after initial symptoms; 4 patients are dead. The average time from initial symptoms to death is 10.6 months (range, 10 weeks to 2 years). All patients were diagnosed by imaging (mostly by CT) and by biopsy. The tumor was located in different brain lobes in 5 patients. Among those, 4 patients had an additional site of tumor involvement, such as the basal ganglia, periventricular area, and right eye. Two patients had involvement of the meninges with epidural tumors of the spinal cord. Of the latter, 1 patient had reported systemic involvement of the bone marrow and the liver at the time of diagnosis.

The flow-cytometric histograms represent the number of cells on the y-axis plotted against the amount of DNA on the $\mathrm{x}$-axis. The first, large peak corresponds to the G0/G1 phase of the DNA cycle. The second, much smaller peak represents the $\mathrm{G} 2 / \mathrm{M}$ phase. The area below the curve between $\mathrm{G} 0 / \mathrm{G} 1$ and $\mathrm{G} 2 / \mathrm{M}$ is the Sphase. The ploidy and SPF of all 11 tumors was recorded (Table 3 and 4). The average SPF for primary intraocular tumors was $23.8 \%$ (range, 18.9 to 29.8 ). Three ocular tumors in cases 1, 2, and 3 had only diploid populations (Fig. 2A) and one eye tumor in case 4 had a near-diploid population (Fig. 2B). The average SPF for primary CNS tumors was $15.1 \%$ (range, 1.1 to 25.1). Five brain tumors had only diploid populations (Fig. 2C) and no abnormal DNA content. Brain tumors in cases 5 and 9 contained aneuploid populations (Figs. $2 \mathrm{D} \& \mathrm{E})$. 


\section{DISCUSSION}

In this study, DNA flow cytometry was performed on 4 primary large B-cell lymphomas of the eye and 7 primary large B-cell lymphomas of the CNS. The DNA content and S-phase fractions (SPF) were measured. Out of the 11 tumors analyzed, 2 brain tumors were aneuploid and 1 eye tumor was peridiploid. All the other tumors were diploid. These results involve too few samples to draw any significant conclusions based solely on DNA content. Furthermore, in the literature, aneuploidy has failed to provide any meaningful clinical significance $(21,22)$.

Regarding tumor proliferation, the average SPF for primary intraocular lymphomas is significantly higher than that of primary CNS lymphomas. This apparent increase in tumor proliferation suggests that primary intraocular lymphoma may be more aggressive and of a higher grade than primary CNS lymphoma. This in turn may explain the clinical observation whereby patients with primary intraocular lymphoma have a higher rate of subsequent CNS involvement as opposed to a lower rate of intraocular involvement in primary CNS lymphoma $(50 \%-80 \%$ vs. $12 \%-18 \%)(1,2,14)$. The primary intraocular lymphoma may thus be considered to have a higher malignant potential. The difference in grade and aggressivity between the two neoplasms could presumably be caused by either environmental or genetic factors.

The intraocular spatial location might be the causative factor stimulating proliferation due to a richer blood supply or the presence of different chemical growth factors. Moreover, the tumor location between the retina and the choroid leads to a unilateral T-cell reaction in which the retinal side of the tumor may be able to grow without immune interference.

Alternatively, the malignant cells of primary intraocular lymphoma might be genetically predisposed to higher proliferation rates. The data presented herein thus raises the possibility that primary CNS and intraocular lymphomas may arise from different progenitor cells. This hypothesis would explain why primary intraocular and CNS lymphomas do not behave like other extranodal non-Hodgkin's lymphomas and why there is no apparent spreading mechanism for neoplastic cells within the CNS and the retina. Support for this theory is found in a recent paper by Alizadeh et al., which demonstrates molecular heterogeneity within diffuse large B-cell lymphomas via gene expression profiling (23).

There are several factors that may have influenced the results of this study. First, the number of tissue samples [11] was small and interpatient variation might have played an important role. Second, although the cell viability is not considered as an important factor in
DNA flow cytometric analysis and the usefulness of paraffin-embedded tissue has been demonstrated (20), fresh tissue samples still yield more accurate results; there is a smaller amount of debris resulting in sharper peaks on flow-histograms. Third, the statistical analysis was not adjusted for all possible interfering factors, such as patient age, gender, precise tumor location, HIV status, and treatment modality. Lastly, intrapatient circadian variation of cell proliferation in nonHodgkin's lymphomas has been demonstrated and may bias the results as well (24).

In conclusion, the results presented in this report suggest that intraocular and CNS lymphoma have different proliferation rates and thus might represent genetically distinct types of neoplasms. Future studies will include DNA flow cytometry on a greater number of tissue samples and genetic profiling using PCR or complementary DNA microarrays (23). Such experiments would represent a further step in the understanding of the genetic identity and pathogenesis of these neoplasms.

\section{ACKNOWLEDGEMENTS}

The author wishes to thank the McGill University Medical Faculty for the Winter Research Bursary and the Cedars Cancer Foundation that provided financial support for this project. Dr. Richardson at the Montreal Neurological Institute for providing primary CNS lymphoma biopsy samples, and Dr. Eduard Tabah at the Royal Victoria Hospital Cancer Registry for allowing access to computerized and manually filed patient data.

\section{REFERENCES}

1. Grant JW, Isaacson PG. Primary Central Nervous System Lymphoma. Brain Pathology 1992;2:97-109.

2. Char DH, Ljung BM, Miller T, Phillips T. Primary Intraocular Lymphoma (ocular reticulum cell sarcoma)

Diagnosis and Management. Ophthalmology 1988;95:625-630.

3. Hen-linger U. Primary CNS lymphoma: Findings outside the brain. Journal ofNeuro-Oncology 1999;43:227-230.

4. Deangelis LM. Current Management of Primary Central Nervous System Lymphoma. Oncology 1995; 9:63-71.

5. Bataille B, Delwail V, Menet E, et al. Primary Intracerebral Malignant Lymphoma: Report of 248 cases. Journal of Neurosurgery 2000;92:261-266.

6. Carvalho AM, Martinu T, Figueiredo AP, Correia CP, Bumier MNJr. Invest Ophthalmol Vis Sci 41 (Suppi): 4, 2000

7. Peterson K, Gordon KB, Heineman MH, DeAngelis LM. The Clinical Spectrum of Ocular Lymphoma. Cancer 1993;72:843-849.

8. Freeman LN, Schachat AP, Knox DL, et al. Clinical Features, Laboratory Investigations, and Survival in Ocular Reticulum Cell Sarcoma. Ophthalmology 1987;94:16311639.

9. Wilson DJ, Braziel R, Rosenbaum JT. Intraocular Lymphoma: Immunopathologic Analysis of Vitreous Biopsy Specimens. Arch Ophthalmol 1992;! 10:1455-1458. 
10. Ljung BM, Char D, Miller TR, Deschenes J. Intraocular Lymphoma: Cytologic Diagnosis and the Role of Immunologic Markers. The International Academy of Cytology Acta Cytologica 1988;32840-847.

11. Lopez JS, Chan CC, Bumier M, et al. Immunohistochemistry Findings in Primary Intraocular Lymphoma. American Journal of Ophthalmology 1991;12:472-474.

12. McLean IW, Bumier MN, Zimmerman LE, Jakobiec FA. Atlas of Tumor Pathology: Tumors of the Eye and Ocular Adnexa. Washington DC: Armed Forces Institute of Pathology, 1993;138-141.

13. Bumier MN, Blanco G. Masquerade Syndrome: Primary Intraocular Lymphoma. In YannofM. Ophthalmology 1998:241-244.

14. Akpek EK, Ahmed I, Hochberg FH, et al. Intraocular-Central Nervous System Lymphoma: Clinical Features, Diagnosis, and Outcomes. Ophthalmology 1999;106:1805-1810.

15. Whitcup SM, de Smet MD, Rubin BI, et al. Intraocular Lymphoma. Ophthalmology 1993;100:1399-1406.

16. Paulus W. Classification, Pathogenesis and Molecular Pathology of Primary CNS Lymphomas. Journal ofNeuro-Oncology 1999,43:203-208

17. Brown MT, Me Clendon RE, Gockerman JP. Primary Central Nervous System Lymphoma with Systemic Metastasis: Case Report and Review. Journal ofNeuro-Oncology 1995,23:207221.

18. Paulus W, Jellinger K. Comparison of integrin adhesion molecules expressed by primary brain lymphomas and nodal lymphomas. Acta Neuropathol 1993;86:360-364.
19. O'Neill BP, Dinapoli RP, Kurtin PJ, Hebemiaim TM. Occult systemic Non-Hodgkin's Lymphoma (NHL) in patients initially diagnosed as Primary Central Nervous System Lymphoma (PCNSL): How much staging is enough? journal ofNeuroOncology 1995;25:67-71.

20. Riley RS, Mahin EJ, Ross W. DNA Ploidy and Cell Cycle Analysis. In: Riley RS, Mahin EJ, Ross W, eds. Clinical Applications of Flow Cytometry. New-York: Igaku-Shoin, 1993;sect.II,chap.6.

21. Riley RS. Clinical Applications of DNA Ploidy and Cell Cycle Analysis. In: Riley RS, Mahin EJ, Ross W, eds. Clinical Applications of Flow Cytometry. New-York: Igaku-Shoin, 1993;sect.II,chap.8.

22. Rehn S, Glimelius B, Sundstrom C. A comparative Study of Proliferation-Associated Parameters in B-cell Non-Hodgkin Lymphoma. Hematological Oncology 1991;9:287-298.

23. Alizadeh AA, Eisen MB, Davis RE, et al. Distinct types of diffuse large B-cell lymphoma identified by gene expression profiling. Nature 2000;403:503-511.

24. Smaaland R, Lote K, Sothem RB, Laerum OD. DNA Synthesis and ploidy in Non-Hodgkin's Lymphomas Demonstrate Intrapatient Variation Depending on Circadian Stage of Cell Sampling. Cancer Research 1993,53:3129-3138.

Tereza Martinu, MD CM, graduated from McGill University School of Medicine in 2002 and completed her Internal Medicine residency at Duke University in Jun 2005. She is starting her Pulmonary and Critical Care Medicine fellowship at Duke University in July 2005. The research presented in this article was completed during her medical school training under the supervision of Dr. Burnier.

Dr. Miguel Burnier, MD, PhD, is currently Chief of Ophthalmology at McGill University Health Centre and at the Jewish General Hospital, as well as chair of the Department of Ophthalmology at McGill University, Montreal. 